\section{Skeletal muscle effects of electrostimulation after COPD exacerbation: a pilot study}

\author{
A. Abdellaoui*,\#, C. Préfaut*, F. Gouzi*, A. Couillard", M. Coisy-Quivy*, G. Hugon*, \\ N. Molinari ${ }^{+, \S}$, T. Lafontaine ${ }^{\#}$, O. Jonquet ${ }^{f}$, D. Laoudj-Chenivesse* and M. Hayot*
}

ABSTRACT: Muscle dysfunction is a major problem in chronic obstructive pulmonary disease (COPD), particularly after exacerbations. We thus asked whether neuromuscular electrostimulation (NMES) might be directly useful following an acute exacerbation and if such a therapy decreases muscular oxidative stress and/or alters muscle fibre distribution.

A pilot randomised controlled study of NMES lasting 6 weeks was carried out in 15 in-patients ( $n=9$ NMES; $n=6$ sham) following a COPD exacerbation. Stimulation was delivered to the quadriceps and hamstring muscles $(35 \mathrm{~Hz})$. Primary outcomes were quadriceps force and muscle oxidative stress.

At the end of the study, quadriceps force improvement was statistically different between groups $(p=0.02)$, with a significant increase only in the NMES group (median (interquartile range) 10 (4.7$11.5) \mathrm{kg} ; \mathrm{p}=0.01$ ). Changes in the 6 -min walking distance were statistically different between groups $(p=0.008)$, with a significant increase in the NMES group (165 $(125-203) m ; p=0.003)$. NMES did not lead to higher muscle oxidative stress, as indicated by the decrease in total protein carbonylation $(p=0.02)$ and myosin heavy chain carbonylation $(p=0.01)$ levels. Finally, we observed a significant increase in type I fibre proportion in the NMES group.

Our study shows that following COPD exacerbation, NMES is effective in counteracting muscle dysfunction and decreases muscle oxidative stress.

KEYWORDS: Chronic obstructive pulmonary disease, exacerbation, fibre type distribution, neuromuscular electrostimulation, oxidative stress

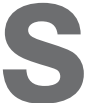
ignificant skeletal muscle dysfunction has been observed during exacerbations of chronic obstructive pulmonary disease (COPD) [1-3]. Several mechanisms may contribute to this effect, such as nutritional imbalance [4], medications [5], physical inactivity [2], systemic inflammation [3] and oxidative stress [6, 7]. It has been suggested that oxidative stress is increased in COPD patients during an acute exacerbation because these patients show increased plasma [6] and skeletal muscle [7] antioxidant capacities. Indeed, oxidative stress is already considered to play a major role in peripheral muscle dysfunction of COPD patients, both at rest and after exercise [8-10]. Oxidative stress affects myofibrillar proteins and especially myosin heavy chain (MHC), which elicits functional and structural changes in muscle type fibres [11, 12].

After COPD exacerbation, the development of muscle dysfunction is rapid, whereas recovery is slow and partial [3]. Early respiratory rehabilitation has been shown to be effective in counteracting this negative progress [1, 13-18]. Direct stimulation of muscle nerves through application of electrical currents using neuromuscular electrostimulation (NMES) has beneficial effects on muscle strength and performance in stable COPD patients [19-22], but has never been used in unstable COPD patients. Since this technique does not induce a ventilatory response and dyspnoea [22], it may be considered as an alternative strategy to increase the muscle work performed in unstable COPD patients. Nevertheless, it remains to be elucidated whether NMES might be useful following an acute exacerbation and if it could stabilise or even decrease muscular oxidative stress in unstable patients.

We thus carried out a pilot study in severe COPD patients during recovery from acute exacerbation to test whether NMES could counteract the deleterious effects of COPD exacerbations. All patients were randomly distributed in two groups.

\section{AFFILIATIONS}

*INSERM U1046 - CHU Montpellier,

${ }^{\S}$ Laboratoire de Biostatistique, EA

2415,

${ }^{\text {R}}$ Réanimation Médicale et Assistance Respiratoire, CHU Montpellier, Montpellier,

${ }^{\#}$ Clinique du Souffle La Vallonie Fontalvie, Lodève,

"Clinique du Souffle La Solane -

Fontalvie, Osséja, and

${ }^{+}$Service de Biostatistique,

Epidemiologie, Santé Publique et Information Médicale, CHU Nîmes, Nîmes, France.

CORRESPONDENCE

A. Abdellaoui

INSERM U1046

Clinique du Souffle La Vallonie Fontalvie

800 avenue Joseph Vallot

34700 Lodève

France

E-mail: aldjia.abdellaoui@inserm.fr

Received:

Oct 262010

Accepted after revision:

Feb 142011

First published online:

Feb 242011 
Our primary objective was to explore whether a 6-week NMES programme could improve muscle strength during recovery of acute COPD exacerbation. Moreover, skeletal muscle biopsies were performed before and after the trial to investigate the effects of NMES on muscle oxidative stress, particularly MHC oxidation. To achieve this objective, we measured protein oxidation (carbonyl formation) and lipid peroxidation (4hydroxy-2-nonenal (4-HNE) protein adduct formation and thiobarbituric acid reactive substances (TBARs)). Lastly, the proportion and composition of fibre type were determined.

\section{MATERIALS AND METHODS}

A detailed description of the methods is available in the online supplementary material.

\section{Study subjects}

Consecutive patients with COPD (forced expiratory volume in one second/forced vital capacity $<70 \%$ ) admitted to the intensive care unit (ICU) of Gui Chauliac hospital, Montpellier, France, for an exacerbation were included in this study. The following criteria were used for patients' selection: 1) diagnosis of acute exacerbation of COPD; 2) age $<75$ yrs; 3 ) body mass index (BMI) $<30 \mathrm{~kg} \cdot \mathrm{m}^{-2}$; 4) no locomotor or neurological condition or disability that could limit the ability to perform exercise; and 5) no pacemaker implantation. All patients gave their written informed consent. The ethics committee Montpellier Sud-Méditerranée-IV approved this study.

\section{Study design and randomisation procedure}

Details on the randomisation procedure are provided in the online supplementary material. Patients were randomly assigned to sham or NMES training. The sham group had weekly therapeutic education sessions, daily active-passive mobilisation and sham electrostimulation. The NMES group followed the same programme, but received effective electrostimulation. The programme started in the ICU, after baseline evaluations, and continued in an in-patient rehabilitation unit (Clinique Souffle Vallonie, Lodève, France).

\section{NMES protocol}

The quadriceps and hamstring muscles of both legs were electrostimulated (Phenix-S8; Vivaltis, Lattes, France) using biphasic symmetric, constant current impulses with a pulse width of $400 \mu \mathrm{s}$ and a frequency of $35 \mathrm{~Hz}$ for $1 \mathrm{~h}$ per day, 5 days per week for 6 weeks. Intensity was set as the maximal tolerable intensity for each patient in the NMES group. The sham group was exposed to the same regimen, except that the stimulation did not cause visible or palpable contractions.

\section{Clinical and functional evaluations}

We assessed the maximal voluntary contraction (MVC) of the quadriceps of each leg and we reported the greatest value. 6min walking test: patients were asked to walk at their own maximal rate without running for $6 \mathrm{~min}$. Patients could stop and restart the test. Symptoms of dyspnoea were assessed using the Medical Research Council (MRC) scale [23].

\section{Muscle biopsy analysis}

Muscle oxidation was assessed by measuring total protein and MHC carbonylation [8, 24] and the level of 4-HNE protein adducts [24] by immunoblotting; lipid peroxidation by measuring TBARs [25].

Fibre typology was evaluated by immunohistochemistry on frozen sections from the muscle biopsies using a panel of antiMHCI (A4.951-c), anti-MHCIIa (2F7-c) and anti-MHCIIx (6H1-c) monoclonal antibodies (University of Iowa, Iowa City, IA, USA) $[26,27]$. Fibre number and size were identified with a microvision image analysis system (Histolab 6.1.0; MicrovisionInstruments, Evry, France).

\section{Statistical analysis}

Data are presented as median and interquartile range in the tables and as box whisker plots in the figures. The MannWhitney nonparametric test was used for comparisons between groups. The Wilcoxon nonparametric paired test was employed to compare different variables at baseline and at the end. Multivariate analysis of variance was employed to compare fibre type distribution in both groups. Spearman's coefficient was used to assess correlations between variables. Bonferronitype adjustments were performed for multiple comparisons. A $p$-value $\leqslant 0.05$ was considered significant. In addition, the effect size was calculated for results that approached significance $(0.05<\mathrm{p}<0.16)$, but not for variables that were significant $(\mathrm{p}<0.05)$. Cohen's conventions for effect size were used for interpretation, where effect size $<0.2,0.5$ and 0.8 are considered as small, medium and large, respectively. Statistical analysis was performed using the statistical package Sygmastat 1.0 (Jandel Scientific, San Rafael, CA, USA).

\section{RESULTS}

\section{Patients}

Of the 47 patients with COPD exacerbation who were initially screened, only 17 were enrolled in the study (fig. S1, online supplementary material). 19 were not included due to the presence of one or more exclusion criteria: unstable ischaemic heart disease $(n=2), B M I>30 \mathrm{~kg} \cdot \mathrm{m}^{-2}(n=5)$, lung cancer $(n=4)$, associated orthopaedic problems $(n=3)$, scheduled surgery $(n=5)$ or inclusion in another protocol $(n=1) .11$ patients declined to participate, citing "fear of biopsy" $(n=3)$, "returning home" $(n=6)$, and family refusal $(n=1)$. Among the 17 included patients, one was later excluded due to readmission to ICU following a new exacerbation and another withdrew consent, $48 \mathrm{~h}$ after inclusion, owing to family refusal. Finally, nine patients in the NMES group and six in the sham group completed the study. All patients received standard treatment, including nebulised bronchodilators, oral or intravenous antibiotics and oral corticosteroids (30-40 mg daily) for 12 weeks. At admission to the ICU, patients were placed under oxygen (three NMES and two sham patients), noninvasive ventilation (three NMES and two sham patients) for 17 days and 20 days, for NMES and sham patients, respectively, or orotracheal intubation with mechanical ventilation (three NMES and two sham patients) for 12 days and 11 days, for NMES and sham patients, respectively. The patients' baseline characteristics (table 1) were similar in the two groups. The NMES programme began on average at $12 \pm 8$ days of hospitalisation in the respiratory ICU.

\section{Tolerance of the NMES sessions}

No adverse effect was recorded during the study. All patients could complete $29 \pm 1$ sessions on average. In the NMES group 


\section{TABLE 1 Patients' baseline characteristics}

\begin{tabular}{lccc} 
Variables & Sham & NMES & p-value \\
\hline Females/males $\mathbf{n}$ & $0 / 6$ & $2 / 7$ & \\
Age yrs & $67(59-72)$ & $59(57-69)$ & 0.74 \\
Weight kg & $56(52-66)$ & $64(52-71)$ & 0.57 \\
BMI kg $\cdot \mathbf{m}^{-2}$ & $19(17-25)$ & $23(17-24)$ & 0.62 \\
FEV $\mathbf{1} \%$ pred & $15(10-27)$ & $25(17-41)$ & 0.16 \\
FVC \% pred & $34(31-54)$ & $53(42-64)$ & 0.28 \\
FEV1/FVC \% pred & $35(31-39)$ & $42(34-52)$ & 0.43 \\
Walking distance $\mathbf{m}$ & $52(0-90)$ & $0(0-135)$ & 0.82 \\
Dyspnoea MRC scale & $5(5-5)$ & $5(5-5)$ & 0.49 \\
MVC kg & $7(3-14)$ & $4(2-9)$ & 0.40 \\
\hline
\end{tabular}

Data are presented as median (interquartile range), unless otherwise stated NMES: neuromuscular electrostimulation; BMI: body mass index; FEV1: forced expiratory volume in $1 \mathrm{~s}$; \% pred: \% predicted; FVC: forced vital capacity; MRC: Medical Research Council; MVC: maximal voluntary contraction.

the intensity of the stimulation was $22 \pm 11 \mathrm{~mA}, 15 \pm 5$ at inclusion and $47 \pm 8 \mathrm{~mA}$ and $32 \pm 1 \mathrm{~mA}$ at the end for quadriceps and hamstring muscles, respectively.

\section{Functional effects of the NMES}

MVC changes were statistically different between groups $(p=0.02)$ (fig. 1a), with a clinically significant increase in the NMES group (median (interquartile range) $10(4.7-11.5) \mathrm{kg}$; $\mathrm{p}=0.01$ ) between inclusion and week 6 (fig. S2A, online supplementary material), whereas only a slight and not significant increase was observed in the sham group (median $3(1-5) \mathrm{kg} ; \mathrm{p}=0.12)$. Changes in the 6-min walking distance were significant between groups $(p=0.008)$ (fig. $1 b)$, with a clinically significant increase in the distance covered by the NMES group (median $165(125-203) \mathrm{m} ; \mathrm{p}=0.03$ ) and only a smaller and not significant improvement in the sham group (median 58 (43-115) m; p=0.24) (fig. S2B, online supplementary material). Finally, the dyspnoea score decreased by two points in the NMES group and by only one point in the sham group.

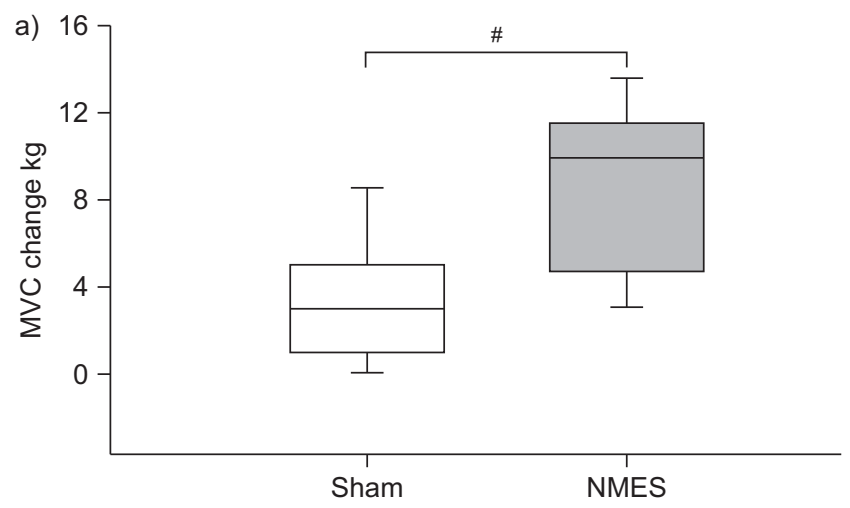

\section{Muscle oxidative stress}

As shown in figure 2a, the anti-DNP antibody detected several protein bands in the quadriceps muscles from patients in the sham and NMES groups. At baseline, the carbonylation levels of total proteins and $\mathrm{MHC}$ were comparable in the two groups (figs S3A and S3B, online supplementary material). At the end of the programme, the changes in carbonyl group formation in total proteins and $\mathrm{MHC}$ were significantly different between groups ( $p=0.02$ and $p=0.04$, respectively) (fig. $2 b$ and $c$ ). Indeed, carbonylation was significantly decreased in the NMES group when compared to baseline values $(p=0.02$ and $p=0.01$, respectively), whereas it remained unchanged in the sham group ( $p=0.21$ and $p=0.62$, respectively) (figs S3A and $\mathrm{S} 3 \mathrm{~B}$, online supplementary material). The carbonylated $\mathrm{MHC} /$ total MHC content ratio was also significantly reduced in the NMES group $(p=0.03)$ at the end of the trial, but did not change in the sham group $(\mathrm{p}=0.40)$. Although the difference in carbonylated $\mathrm{MHC} /$ total $\mathrm{MHC}$ content ratio were not significantly different between groups, the effect size tended to be large (effect size 1.09; $\mathrm{p}=0.08$ ).

The level of 4-HNE protein adducts and lipid peroxidation (data not shown) did not differ between groups at baseline and remained unmodified after the programme (fig. S4A and S4B, online supplementary material). However, the effect size for 4HNE protein levels tended to be medium (effect size 0.4; $\mathrm{p}=0.1$ ), that could be considered as a moderate effect.

The levels of $\mathrm{Cu} / \mathrm{Zn}$-superoxide dismutase, glutathione reductase and catalase antioxidant enzymes did not significantly change in either group (fig. 3). The effect size for the antioxidant enzymes levels was not calculated because all pvalues were $>0.16$.

\section{Muscle structure}

No significant differences were found at baseline between groups (table 2). Changes in the proportion of type I and IIa/ IIx fibres were significantly different between groups $(p=0.03$ and $p=0.04$, respectively) at the end of the study. The proportion of type I and IIa/IIx fibres significantly increased (from $13 \pm 4 \%$ to $25 \pm 3 \% \quad(p=0.002)$ and $9 \pm 2 \%$ to $23 \pm 4 \%$ $(\mathrm{p}=0.003)$, respectively) in the NMES group, whereas they remained unchanged in the sham group (table 2). A significant

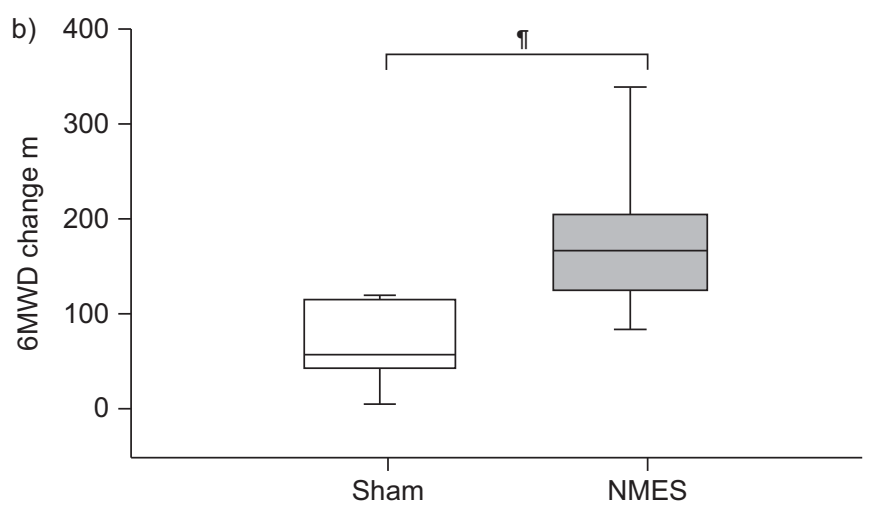

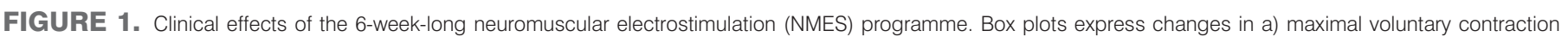

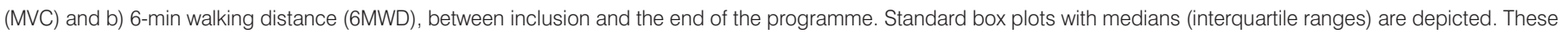
changes were statistically different between groups. ${ }^{*}: p=0.02 ;{ }^{\bullet}: p=0.008$. 
a)

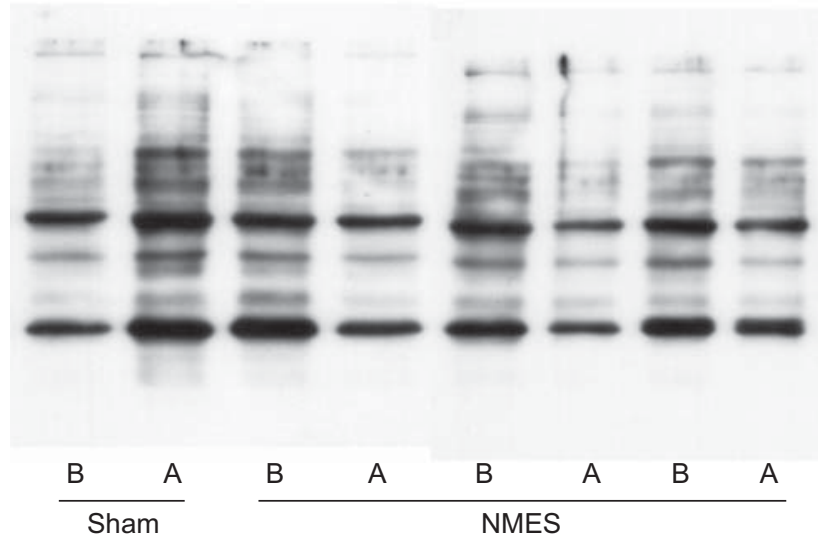

c)

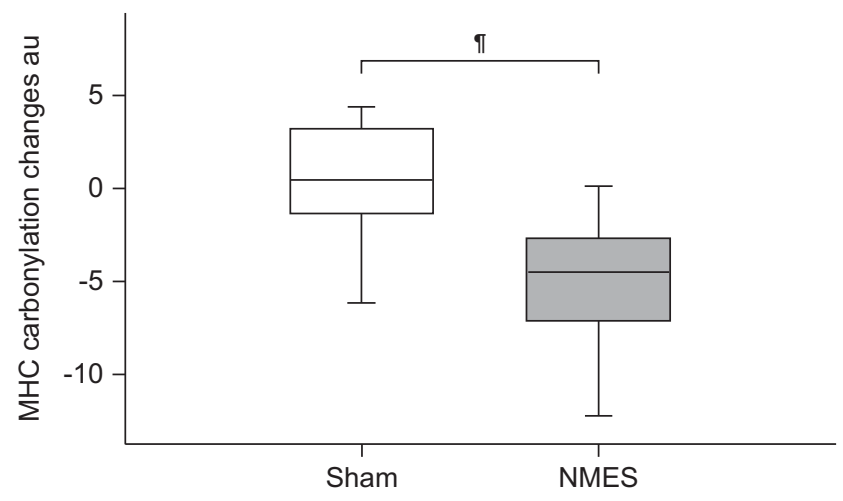

b)

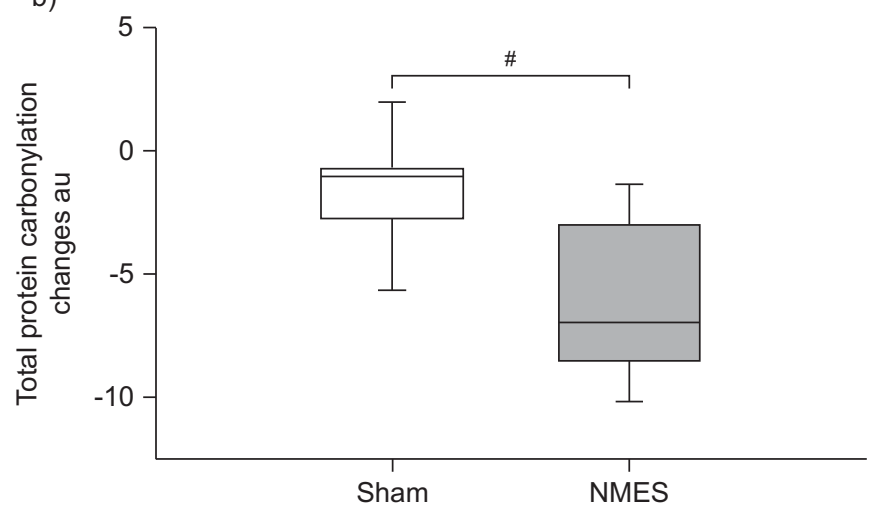

FIGURE 2. Protein carbonylation. a) Representative examples of carbonylated proteins in the vastus lateralis muscle from patients in the sham and neuromuscular electrostimulation (NMES) groups, before (B) and after (A) the programme. Carbonylated proteins of different molecular weights were detected. Quantification of the changes in carbonylation (detected as variations in optical density) of b) total proteins and of $\mathrm{c}$ ) myosin heavy chain ( $\mathrm{MHC}$ ) in the sham and NMES groups. Data are described using standard box plots with medians (interquartile ranges). Total protein and $\mathrm{MHC}$ carbonylation changes were significantly different in the two groups, at the end of programme. ${ }^{*}: p=0.02 ;{ }^{\circ}: p=0.04$.

negative correlation was observed between changes in the proportion of type I fibres and variation of carbonylated MHC $(\mathrm{r}=-0.60 ; \mathrm{p}=0.03) \quad$ (fig. 4a). Although the changes in the proportion of IIx fibres were not significantly different between groups, a significant decrease in the proportion of type IIx fibres in both groups was observed (table 2).

The size of type I but not of type IIx fibres was significantly increased in the NMES group at the end of the programme $(\mathrm{p}=0.009$ and $\mathrm{p}=0.16$, respectively), whereas it remained unchanged in the control group (data not shown).

Finally, the changes in total MHC content were statistically different between groups $(p=0.03)$. Moreover, a significant positive correlation was found between changes in $\mathrm{MHC}$ content and changes in quadriceps strength $(r=0.79, \mathrm{p}<0.001)$ (fig. 4b).

\section{DISCUSSION}

The present study shows that during recovery from acute exacerbation, a 6-week training protocol using NMES induced: 1) improvement of the muscle force and distance covered during the walking test; 2) reduction of total protein carbonylation, and particularly of carbonylated $\mathrm{MHC}$; 3) increase in MHC content; and 4) significant changes in muscle fibre typology in the NMES group in comparison to control patients.

We chose to study patients after COPD exacerbation because it has been shown this represents the best model of extreme muscle dysfunction. Indeed, SPRUIT et al. [3] have clearly

demonstrated lower peripheral muscle force in patients after hospitalisation for acute COPD exacerbation than in patients with stable COPD. Moreover, the average quadriceps strength in all our patients at inclusion was extremely weak (MVC $<10 \mathrm{~kg}$ ) and was much lower than the value usually observed in stable patients [28]. In addition, skeletal muscle protein oxidation was more important in our patients than in patients with stable COPD (unpublished data). Furthermore, according to the meta-analysis by GOSKER et al. [29], a proportion of type I fibres $<27 \%$, as described in our study $(13 \%)$, is abnormally low. Likewise, it has been proposed that a proportion of type IIx fibres $>29 \%$, also observed in our patients at inclusion (39\%), should be considered as abnormally high [29].

\section{Functional effects of the NMES programme}

The NMES group presented a more important and significant increase of quadriceps strength that the sham group at the end of the 6-week programme. Although the results were not significant, a slight spontaneous recovery of quadriceps strength occurred in the sham group, which was probably due to the reversibility of the systemic effects of acute COPD exacerbation and the daily mobilisation by a physiotherapist. This result is consistent with the study by SPRUIT et al. [3]. These authors reported that, 90 days after hospitalisation, the quadriceps peak torque was only increased by a mean of $8 \%$ in comparison to the value at day 8 after exacerbation.

The distance covered during the walking test significantly increased in the NMES group, whereas in the sham group the increase was small at the end of the 6-week programme. 
a)
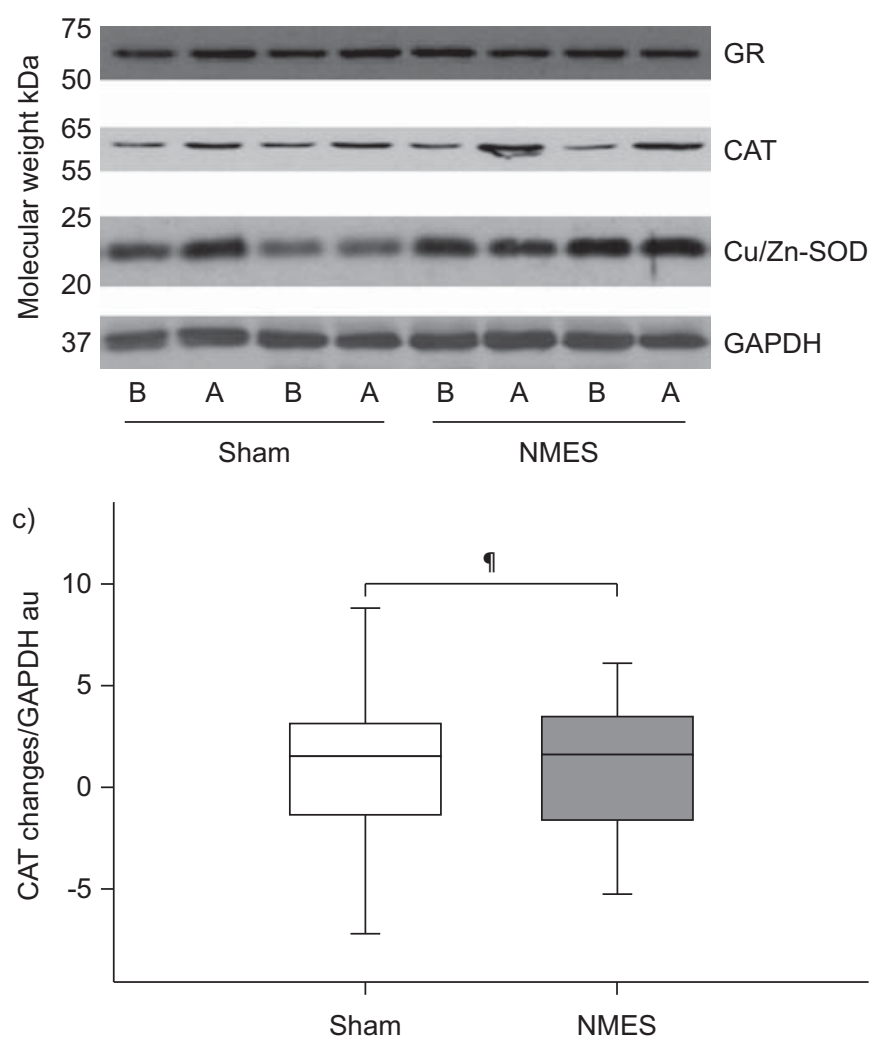
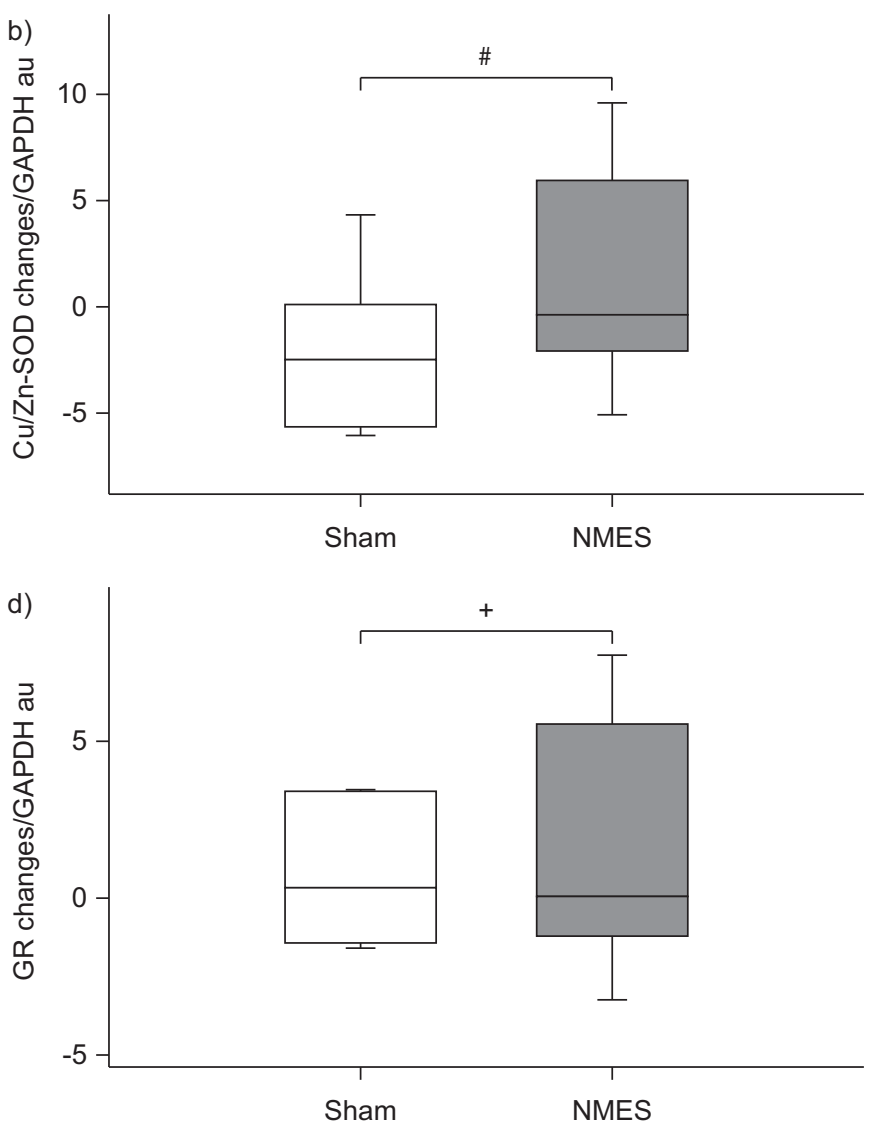

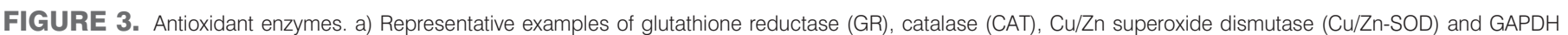

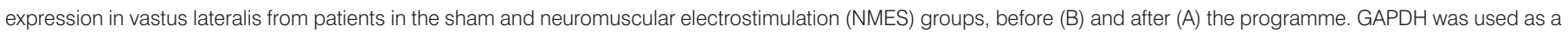

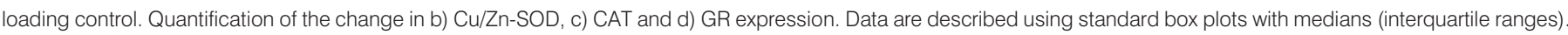
Changes in the expression of antioxidant enzymes did not differ in the two groups. ${ }^{*}: p=0.20 ;{ }^{\bullet}: p=0.92 ;{ }^{+}: p=0.55$.

This result is consistent with the study by MAN et al. [17] who have shown that, 3 months after exacerbation, patients in the group of usual care had decreased exercise capacity. The metaanalysis by PUHAN et al. [13] has highlighted a significantly improved exercise capacity after early rehabilitation. A recent interesting study showed that outpatient pulmonary rehabilitation immediately following an acute COPD exacerbation

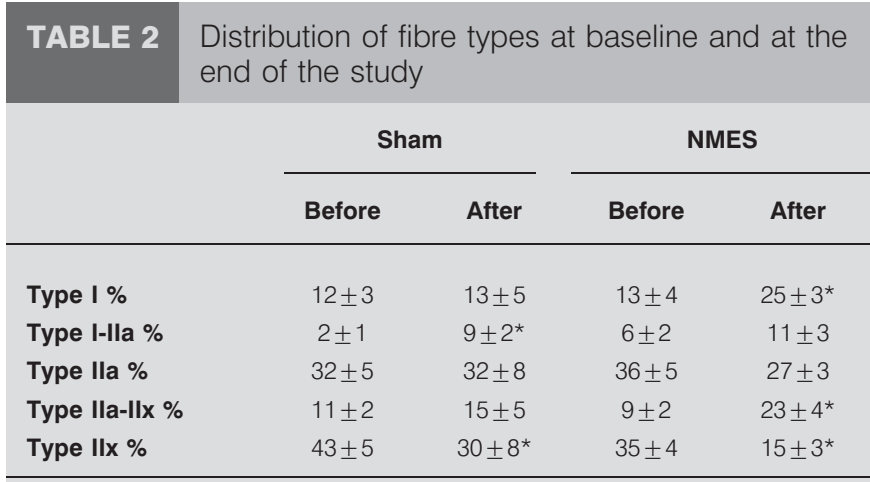

Date are presented as mean \pm SEM. NMES: neuromuscular electrostimulation *: significantly different between before and after the programme. improves exercise capacity partly through increase of quadriceps strength [18].

Moreover, the MRC dyspnoea score already started to decrease at the beginning of the programme in the NMES group, concurrently with the improvement in the walking test. Finally, in our study, we did not report the influence of NMES on the respiratory system, but other authors have observed decreased resting respiratory rate in bed-ridden patients, after NMES training [21] and a small but significant reduction in the ventilation dead space was found at peak exercise in treated individuals in comparison with the sham group [19]. These preliminary findings about the possible impact of NMES on the ventilatory response to exercise require confirmation.

In summary, the NMES programme seems to be an effective strategy to facilitate muscle function recovery in COPD patients following ICU hospitalisation for exacerbation. Nevertheless, the underlying molecular mechanisms of such clinical and physiological improvements remain to be elucidated.

\section{Muscle redox balance}

Our programme significantly decreased the level of carbonylated proteins in muscle of the NMES group in comparison to the sham group and did not increase the level of 4-HNE 

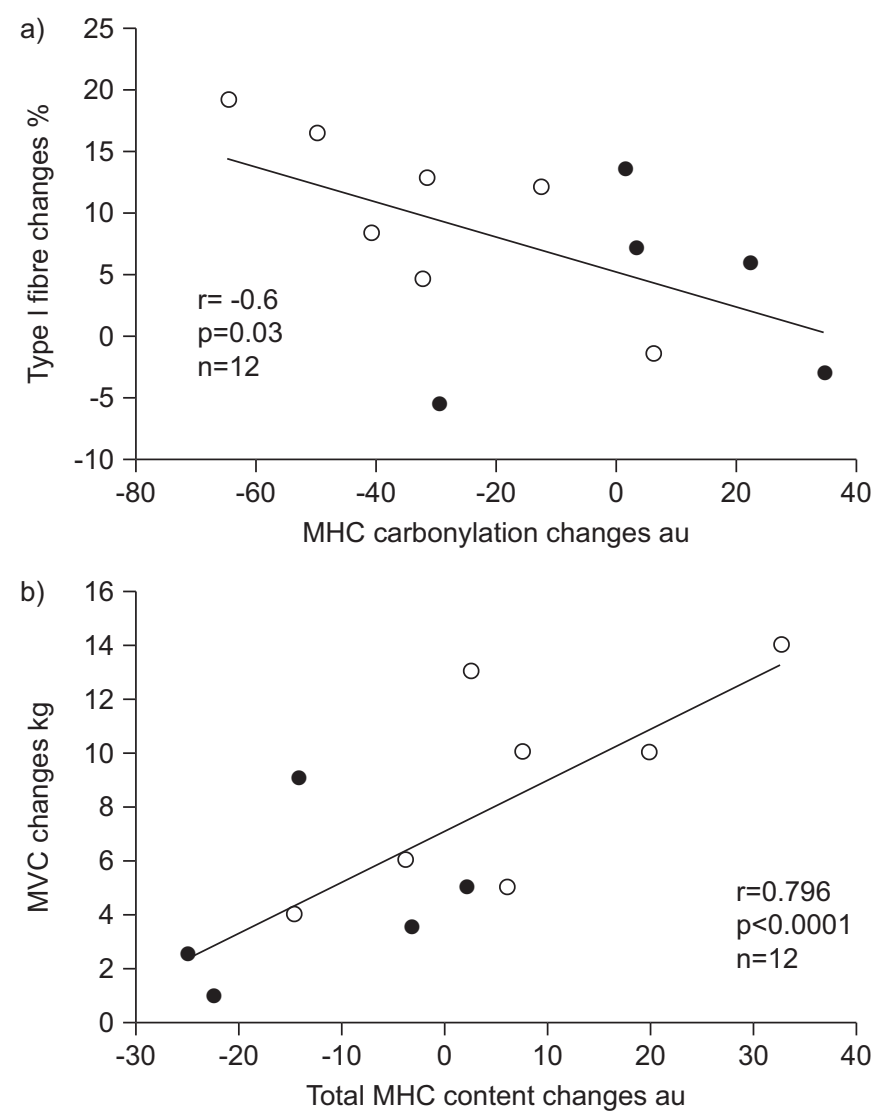

FIGURE 4. Muscle structure. a) The changes in carbonylated myosin heavy chain $(\mathrm{MHC})$ levels, expressed as optical density in arbitrary units, were inversely correlated with the changes in the proportion of type I fibres. b) The increase in total MHC content, expressed as optical densities in arbitrary units, was positively correlated with improvement in the quadriceps maximal voluntary contraction (MVC).

- : sham group; $\bigcirc$ : neuromuscular electrostimulation patient group.

protein adducts and lipid peroxidation. This suggests that localised electrostimulation produces muscle contractions of sufficient intensity to induce functional improvement without enhancing oxidative stress within the muscle. This is an important finding, since patients with COPD exhibit increased muscle oxidative stress, not only after high intensity training [10], but also at rest [8]. The 6-week NMES induced, in particular, a decrease of MHC carbonylation. MARIN-CORRAL et al. [30] reported that, in the diaphragm of severe COPD patients, highly carbonylated MHC is likely to be degraded by the ubiquitin-proteasome pathway. Consequently, although the decrease of carbonylated MHC and increase in MHC content in our patients could not be causally related, it is tempting to suggest that the reduction in carbonylated MHC could permit, at least partly, the increase of MHC content. Conversely, it has been proposed that MHC oxidation by reactive oxygen species may perturb myosin structure and impair myosin function [12]. Accordingly, we observed a correlation between changes in total MHC content and changes in quadriceps strength $(\mathrm{r}=0.79, \mathrm{p}<0.001)$. Altogether, our results suggest that NMES increases muscle strength, probably due to a reduction of the level of MHC oxidation and, therefore, by increasing the quantity of functional $\mathrm{MHC}$ in skeletal muscle. However, future work should explore the exact contribution of oxidative stress to the increase in muscle protein breakdown and myosin loss observed in the quadriceps of COPD patients.

\section{Muscle structure}

Our study is the first to report changes of muscle fibre types in patients with severe COPD after NMES. Consistent with the results obtained in healthy males [31], paraplegic males [32] and chronic heart failure patients [33] after an electrostimulation programme, we observed an increase in type I fibre proportion and a decrease in type IIx fibre proportion in COPD patients after exacerbation. These changes are usually interpreted as transition from fast-to-slow fibre type. Interestingly, we observed a significant correlation between the changes of type I fibre proportion and of carbonylated MHC level $(p=0.03, r=-0.60)$. It is thus tempting to suggest that the gradual shift in the content of MHC isoforms in COPD patients might be due to a decrease of MHC-I carbonylation and, consequently, of its degradation.

However, the signalling pathways involved in the control of fibre type transition are still unknown. Recently, peroxisome proliferator-activated receptor-coactivator-1 (PGC-1 $\alpha$ ) was implicated in the formation of oxidative muscle fibres in mice [34]. Moreover, it has been reported that in COPD muscles, the proportion of oxidative fibres is reduced [29] and the level of PGC-1 $\alpha$ mRNA is lower [35]. Therefore, it would be interesting to test whether the higher amount of type I fibres observed in the NMES group could be induced by up-regulation of PGC- $1 \alpha$.

\section{Study limitations}

One possible critique of our study is the small sample size. However, the retrospectively calculated statistical power of the primary outcome (quadriceps force) was of $96 \%$ and significant results were found despite the relatively small population. Furthermore, finding patients with COPD exacerbation who accepted to be included in a study that included two muscle biopsies was challenging, but offered the opportunity to study biochemical and morphological changes of muscle over time in the same patients. However, it is necessary to conduct a clinical study with more patients to confirm our results. In the literature, there is only one example of a similar project, but only one biopsy was carried out at the end of the programme [15]. We believe that our study, as the previous study [15], provides new information for the management of unstable COPD patients.

Given our results and all the existing studies [1, 14, 15, 17, 18] on the effects of early rehabilitation on muscle function and exercise capacity in unstable patients, NMES, not requiring any patient cooperation, may be considered as an alternative to the standard training method used during the first days of hospitalisation following exacerbation. However, when the patient becomes more autonomous, the standard training modality will be able to enhance and potentiate the effects of the NMES. Therefore, further studies are needed to define if the combination of NMES and standard training methods in COPD patients after exacerbation may lead to a greater improvement of overall patient clinical functions than standard training alone. 
In conclusion, the application of an NMES programme following exacerbation of COPD is an effective strategy to counterbalance the loss of skeletal muscle function. In our patients, it effectively restored muscle function by decreasing oxidative stress and, particularly, MHC oxidation, and by improving MHC content and distribution of type I muscle fibres. We believe that the decrease in muscle oxidative stress, together with the significant changes in muscle fibre typology, may serve as a basis for designing future studies, where electrical stimulation may be used as a muscle training modality in a larger population of unstable COPD patients.

\section{SUPPORT STATEMENT}

This study was supported by grants from the patients' association APARD. A. Abdellaoui and F. Gouzi were supported by a "CIFRE grant" (Conventions Industrielles de Formation par la Recherche) from the Fontalvie Corporation, Toulouges, France, and the French "Ministère délégué à la recherche et aux nouvelles technologies".

\section{CLINICAL TRIAL}

This study is registered at clinicaltrials.gov with identifier number NCT01167283.

\section{STATEMENT OF INTEREST}

Statements of interest for C. Préfaut and M. Hayot can be found at www.erj.ersjournals.com/site/misc/statements.xhtml

\section{ACKNOWLEDGEMENTS}

The authors gratefully acknowledge A. Bouret, G. Carnac and A. Turki (au INSERM U1046 - CHU, Montpellier, France) for their technical support in the laboratory, F. Pillard (INSERM U1046 - CHU) and N. Heraud (Fontalvie Corporation, Toulouges, France) for statistical advice and E. Andermarcher (INSERM U1046 - CHU) for critical reading of the manuscript. M. Desplan (Fontalvie Corporation) is acknowledged for his help with muscle biopsies. The clinical teams of the Respiratory Division (particularly the groups directed by P. Massanet and A. Bourdin) and the teams of the Respiratory Rehabilitation Clinic La Vallonie, Lodève, France were instrumental in assisting with the logistics of the study.

\section{REFERENCES}

1 Puhan MA, Scharplatz M, Troosters T, et al. Respiratory rehabilitation after acute exacerbation of COPD may reduce risk for readmission and mortality: a systematic review. Respir Res 2005; 6: 54 .

2 Pitta F, Troosters T, Probst VS, et al. Physical activity and hospitalization for exacerbation of COPD. Chest 2006; 129: 536-544.

3 Spruit MA, Gosselink R, Troosters T, et al. Muscle force during an acute exacerbation in hospitalised patients with COPD and its relationship with CXCL8 and IGF-I. Thorax 2003; 58: 752-756.

4 Creutzberg EC, Wouters EF, Vanderhoven-Augustin IM, et al. Disturbances in leptin metabolism are related to energy imbalance during acute exacerbations of chronic obstructive pulmonary disease. Am J Respir Crit Care Med 2000; 162: 1239-1245.

5 Decramer M, Lacquet LM, Fagard R, et al. Corticosteroids contribute to muscle weakness in chronic airflow obstruction. Am J Respir Crit Care Med 1994; 150: 11-16.

6 Rahman I, Skwarska E, MacNee W. Attenuation of oxidant/ antioxidant imbalance during treatment of exacerbations of chronic obstructive pulmonary disease. Thorax 1997; 52: 565-568.

7 Crul T, Testelmans D, Spruit MA, et al. Gene expression profiling in vastus lateralis muscle during an acute exacerbation of COPD. Cell Physiol Biochem 2010; 25: 491-500.

8 Couillard A, Maltais F, Saey D, et al. Exercise-induced quadriceps oxidative stress and peripheral muscle dysfunction in patients with chronic obstructive pulmonary disease. Am J Respir Crit Care Med 2003; 167: 1664-1669.

9 Koechlin C, Couillard A, Simar D, et al. Does oxidative stress alter quadriceps endurance in chronic obstructive pulmonary disease? Am J Respir Crit Care Med 2004; 169: 1022-1027.

10 Barreiro E, Rabinovich R, Marin-Corral J, et al. Chronic endurance exercise induces quadriceps nitrosative stress in patients with severe COPD. Thorax 2009; 64: 13-19.

11 Barreiro E, Hussain SN. Protein carbonylation in skeletal muscles: impact on function. Antioxid Redox Signal, 12: 417-429.

12 Yamada T, Mishima T, Sakamoto M, et al. Oxidation of myosin heavy chain and reduction in force production in hyperthyroid rat soleus. J Appl Physiol 2006; 100: 1520-1526.

13 Puhan M, Scharplatz M, Troosters T, et al. Pulmonary rehabilitation following exacerbations of chronic obstructive pulmonary disease. Cochrane Database Syst Rev 2009; 1: CD005305.

14 Clini EM, Crisafulli E, Costi S, et al. Effects of early inpatient rehabilitation after acute exacerbation of COPD. Respir Med 2009; 103: 1526-1531.

15 Troosters T, Probst VS, Crul T, et al. Resistance training prevents deterioration in quadriceps muscle function during acute exacerbations of chronic obstructive pulmonary disease. Am J Respir Crit Care Med 2010; 181: 1072-1077.

16 Clini E, Roversi P, Crisafulli E. Early rehabilitation: much better than nothing. Am J Respir Crit Care Med 2010; 181: 1016-1017.

17 Man WD, Polkey MI, Donaldson N, et al. Community pulmonary rehabilitation after hospitalisation for acute exacerbations of chronic obstructive pulmonary disease: randomised controlled study. BMJ 2004; 329: 1209.

18 Seymour JM, Moore L, Jolley CJ, et al. Outpatient pulmonary rehabilitation following acute exacerbations of COPD. Thorax 2010; 65: 423-428.

19 Bourjeily-Habr G, Rochester CL, Palermo F, et al. Randomised controlled trial of transcutaneous electrical muscle stimulation of the lower extremities in patients with chronic obstructive pulmonary disease. Thorax 2002; 57: 1045-1049.

20 Neder JA, Sword D, Ward SA, et al. Home based neuromuscular electrical stimulation as a new rehabilitative strategy for severely disabled patients with chronic obstructive pulmonary disease (COPD). Thorax 2002; 57: 333-337.

21 Zanotti E, Felicetti G, Maini M, et al. Peripheral muscle strength training in bed-bound patients with COPD receiving mechanical ventilation: effect of electrical stimulation. Chest 2003; 124: 292-296.

22 Vivodtzev I, Pepin JL, Vottero G, et al. Improvement in quadriceps strength and dyspnea in daily tasks after 1 month of electrical stimulation in severely deconditioned and malnourished COPD. Chest 2006; 129: 1540-1548.

23 Bestall JC, Paul EA, Garrod R, et al. Usefulness of the Medical Research Council (MRC) dyspnoea scale as a measure of disability in patients with chronic obstructive pulmonary disease. Thorax 1999; 54: 581-586.

24 Barreiro E, Gea J, Corominas JM, et al. Nitric oxide synthases and protein oxidation in the quadriceps femoris of patients with chronic obstructive pulmonary disease. Am J Respir Cell Mol Biol 2003; 29: 771-778.

25 Ohkawa H, Ohishi N, Yagi K. Assay for lipid peroxides in animal tissues by thiobarbituric acid reaction. Anal Biochem 1979; 95: 351-358.

26 Lucas CA, Kang LH, Hoh JF. Monospecific antibodies against the three mammalian fast limb myosin heavy chains. Biochem Biophys Res Commun 2000; 272: 303-308.

27 Eliason G, Abdel-Halim S, Arvidsson B, et al. Physical performance and muscular characteristics in different stages of COPD. Scand J Med Sci Sports 2009; 19: 865-870.

28 Serres I, Gautier V, Varray A, et al. Impaired skeletal muscle endurance related to physical inactivity and altered lung function in COPD patients. Chest 1998; 113: 900-905. 
29 Gosker HR, van Mameren H, van Dijk PJ, et al. Skeletal muscle fibre-type shifting and metabolic profile in patients with chronic obstructive pulmonary disease. Eur Respir J 2002; 19: 617-625.

30 Marin-Corral J, Minguella J, Ramirez-Sarmiento AL, et al. Oxidised proteins and superoxide anion production in the diaphragm of severe COPD patients. Eur Respir J 2009; 33: 1309-1319.

31 Nuhr M, Crevenna R, Gohlsch B, et al. Functional and biochemical properties of chronically stimulated human skeletal muscle. Eur J Appl Physiol 2003; 89: 202-208.

32 Crameri RM, Weston A, Climstein M, et al. Effects of electrical stimulation-induced leg training on skeletal muscle adaptabi- lity in spinal cord injury. Scand J Med Sci Sports 2002; 12: 316-322.

33 Nuhr MJ, Pette D, Berger R, et al. Beneficial effects of chronic lowfrequency stimulation of thigh muscles in patients with advanced chronic heart failure. Eur Heart J 2004; 25: 136-143.

34 Lin J, Wu H, Tarr PT, et al. Transcriptional co-activator PGC-1 alpha drives the formation of slow-twitch muscle fibres. Nature 2002; 418: 797-801.

35 Remels $\mathrm{AH}$, Schrauwen $\mathrm{P}$, Broekhuizen $\mathrm{R}$, et al. Peroxisome proliferator-activated receptor expression is reduced in skeletal muscle in COPD. Eur Respir J 2007; 30: 245-252. 